Sitzungsber. Abt. II (2005) 214: 161-180 Sitzungsberichte

Mathematisch-naturwissenschaftliche Klasse Abt. II

Mathematische, Physikalische und Technische Wissenschaften

○) Österreichische Akademie der Wissenschaften 2006

Printed in Austria

\title{
Josephson Junction Revisited
}

\author{
By \\ Heide Narnhofer \\ (Vorgelegt in der Sitzung der math.-nat. Klasse am 17. November 2005 \\ durch das w. M. Walter Thirring)
}

\begin{abstract}
In the framework of the BCS Hamiltonian we construct for the ground state in an appropriate scaling a macroscopic non-Abelian algebra, that reflects the behaviour of the Josephson current. For finite temperature it is possible to construct the same algebra but not as a limit of Gibbs states. We have to assume a coherent superposition of the different phases.
\end{abstract}

PACS number: $03.75 \mathrm{Lm}$.

Key words: Josephson effect, coherence.

\section{Introduction}

The Josephson current between two weakly coupled superconductors is a well established fact. It is of special interest because it shows quantum effects on a macroscopic level. In [1] it was predicted as a consequence of the description of superconductors by Cooper pairs and the possibility of tunneling of these Cooper pairs. The calculation was based on perturbation theory. As a consequence the effect of fluctuations was not estimated. Experiments support that these fluctuations are unable to destroy the effect.

After the discovery of the Josephson effect the BCS-model that was the basis of Josephson's considerations was studied in more detail and more rigorousity [2, 3], also in context with the Josephson current $[4,5]$. The mean field structure of the interaction implies other 
features with respect to phase transition and time evolution than we are accustomed to for short range interaction. On the basis of the quasi local algebra, i.e. if only correlations between few Cooper pairs are considered, the phase occurring in the Josephson effect appears as a classical variable in the center of the representation, whereas the time evolution is only well defined in the pure (gauge breaking) phases and there depends on the phase. Therefore the quantum behavior of the phase can only be observed if we extend the class of operators that are described by our state.

Such an extension to a larger class of operators was suggested in [6]. The state is extended to the fluctuation algebra. In this fluctuation algebra individual operators are combined to equivalence classes, if they only differ locally. But with an appropriate scaling they keep a quantum structure that reduces to the one of a Weyl algebra in an appropriate $L^{2}\left(R^{\nu}\right)$ space. In [7] the construction of the fluctuation algebra was extended to systems with mean field interaction and it was shown that new features with respect to time evolution appear. The time evolution of the fluctuation algebra reflects the stability behavior that is fixed by the long range effect of the mean field interaction. Therefore it is natural to examine, whether the Josephson effect can be formulated as a state over a fluctuation algebra. We will see, this is true only to a limited extent.

If we formulate the fluctuation algebra together with its time evolution in the pure (gauge breaking) phases, then the periodicity, characteristic to the Josephson current, disappears. The state over the fluctuation algebra is also not invariant under the time evolution. Therefore the pure states over the quasilocal algebra are of no physical relevance.

We first start with considering the ground state. We can consider statistical and coherent superpositions of the pure states. On the quasilocal level this difference cannot be observed. But for the construction of a phase algebra that replaces the fluctuation algebra of [6] only the coherent superposition can be used. On one hand only the coherent superposition enables us to define the appropriate limit of operators such that in the limit of infinitely extended systems their expectation values coincide with those of the Weyl algebra over the one-dimensional torus. On the other hand only the coherent superposition describes the ground state for a finite system. Extending the construction to two weakly coupled superconductors we obtain the tensor product of two Weyl algebras over one-dimensional tori, one of which reflects the algebra of the Josephson current. We have to note, however, that the appropriate scaling of the interaction between the 
two superconductors is smaller than we expected. As a result the time scale of the Josephson current is of macroscopic scale. The periodicity parameter however is recovered and independent of the strength of the interaction.

If we pass to temperature states we keep the same structure on the quasilocal level as long as we are below phase transition. Again we can construct a coherent superposition of the pure thermodynamical phases. However we have to keep in mind that these phases themselves correspond to density matrices, so that the coherent superposition can only be done in the extended GNS-representation. The construction of the phase algebra can be carried through in analogy to the ground state, however the momentum conjugated to the phase cannot be obtained as the limit of observables, we have to subtract the fluctuations in the form of a regularization with an unphysical operator of the commutant. As soon as we are only interested in commutators with the phase, the effect of this unphysical operator disappears, but this works only on the linear level, i.e. for the Josephson current but not for the total phase algebra. We have offered a rather special sequence of states over the finite systems. We cannot prove that this sequence coincides with the sequence of Gibbs states. But also other sequences would lead to a state of the Weyl algebra over the onedimensional torus, but with an operator that has been renormalized and allows only limited physical interpretation. A sequence of states where no renormalization is necessary definitely differs from the sequence of Gibbs states.

Next we couple two superconductors in order to reproduce the Josephson effect. Again we have to renormalize the spins in order to obtain the conjugated momenta. However for the difference of the momenta (the part responsible for the Josephson effect) it is possible to construct a sequence of states that reproduces the uncoupled state on the quasilocal level but couples the commutants in the GNS representation in such a way that the momentum for the Josephson algebra is obtained as limit of operators without renormalization. Physically this means that the fluctuations in the two superconductors are strongly correlated. Evidently this state is not the limit of Gibbs states. Whether this state can be justified by assuming a coupling between the two superconductors as proposed by Josephson who had to calculate up to second order including a change of the state, and then use the macroscopic scaling in the phase algebra as justification that the actual fluctuations are averaged out in long microscopic time remains an open problem. In addition we notice that the Weyl algebra permits inequivalent representations, that in fact are realized in 
experiment by the adjustment of a voltage. We have not succeeded to find a justification for the connection of this voltage with the representation of the Weyl algebra and have to leave also this problem for further investigation.

In the last chapter we demonstrate that for several strongly coupled superconductors the algebra responsible for the Josephson current is uneffected from the number of superconductors. Strongly coupled superconductors are oriented homogeneously and again just one phase is coherently superposed. Fluctuations between the superconductors are present, but they do not effect the Josephson current, as it is supported by experiment.

\section{The BCS Model}

Based on the considerations about the electron-phonon interaction the Hamiltonian of a superconductor can be written as

$$
H=\sum_{k}^{N} \mu\left(a_{k}^{\dagger} a_{k}+b_{k}^{\dagger} b_{k}\right)-\frac{1}{N} \sum_{k, l}^{N} a_{l} b_{-l} b_{k}^{\dagger} a_{-k}^{\dagger},
$$

where $a_{k}, b_{k}$ are annihilation operators with spin up respectively down and momentum $k$ or $l$ in the neighborhood of the Fermi surface so that the corresponding energy can be considered as independent of $k$. Combining $a_{k} b_{-k}$ the operators for different $k$ become commutative and for the same $k$ can be represented by $\sigma$-matrices in a twodimensional subspace. Therefore we can write the Hamiltonian in the form

$$
\begin{aligned}
H_{B C S} & =-\frac{4}{N} \sum_{k, l=1}^{N}\left(\sigma_{x}^{k} \sigma_{y}^{l}+\sigma_{y}^{k} \sigma_{y}^{l}\right)+\sum_{k=1}^{N} 4 \mu \sigma_{z}^{k} \\
& =-\frac{4}{N} \sum_{k, l=1}^{N}\left(\sigma_{+}^{k} \sigma_{-}^{l}\right)+4 \sum_{k=1}^{N}\left(\mu-\frac{1}{N}\right) \sigma_{z}^{k} .
\end{aligned}
$$

With $S_{z}=\sum_{k=1}^{N} \sigma_{z}^{k}$ the Hamiltonian becomes

$$
H_{B C S}=4\left(\mu-\frac{1}{N}\right) S_{z}-\frac{4}{N} S_{+} S_{-}=4 \mu S_{z}-\frac{4}{N}\left(S^{2}-S_{z}^{2}\right) .
$$

The ground state is obtained by maximizing $S$, i.e. by a vector $\left|\Omega_{N}\right\rangle$ with

$$
\left\langle\Omega_{N}\left|S^{2}\right| \Omega_{N}\right\rangle=\frac{N(N+2)}{4}
$$


and by minimizing with respect to $S_{z}$, i.e. with $\left\langle\Omega_{N}\left|S_{z}\right| \Omega_{N}\right\rangle=-[N \mu / 2]$, where $[N \mu / 2]$ is the integer number closest to $N \mu / 2$, provided $|\mu| \leq 1$. Otherwise $\left\langle\Omega_{N}\left|S_{z}\right| \Omega_{N}\right\rangle=N / 2$ and we have no superconductivity. In the limit $N \rightarrow \infty$ we reach a ground state with expectation values for typical local operators

$$
\left\langle\sigma_{x}^{l}\right\rangle=\left\langle\sigma_{y}^{l}\right\rangle=0, \quad\left\langle\sigma_{z}^{l}\right\rangle=\frac{\mu}{2}=\frac{1}{2} \cos \alpha, \quad\left\langle\sigma_{x}^{l} \sigma_{x}^{k}\right\rangle=\frac{1}{8} \sin ^{2} \alpha .
$$

On the quasilocal algebra this state is not pure but can be decomposed via the central decomposition into the Bogoliubov states $\omega_{\phi}$

$$
\begin{aligned}
\omega & =\int_{0}^{2 \pi} d \phi \omega_{\phi}, \\
\omega_{\phi}\left(\sigma_{z}\right)=\frac{1}{2} \cos \alpha, \quad \omega_{\phi}\left(\sigma_{x}\right) & =\frac{1}{2} \sin \alpha \cos \phi, \quad \omega_{\phi}\left(\sigma_{y}\right)=\frac{1}{2} \sin \alpha \sin \phi .
\end{aligned}
$$

The vector that implements $\omega_{\phi}$ is the limit of product states $\left|\Omega_{\phi, N}\right\rangle=$ $\otimes_{k}^{N}|\alpha, \phi\rangle$. Their coherent superposition implements the ground state:

$$
\left|\Omega_{N}\right\rangle=\left|\int_{0}^{2 \pi} d \phi \Omega_{\phi, N}\right\rangle
$$

This follows because

$$
\begin{aligned}
\exp \left[i \alpha S_{z}-\frac{\mu N}{2}\right]\left|\Omega_{N}\right\rangle & =\left|\int_{0}^{2 \pi} d \phi \Omega_{\phi+\alpha, N}\right\rangle=\left|\Omega_{N}\right\rangle, \\
\exp \left[i a S^{2}\right]\left|\Omega_{\phi, N}\right\rangle & =\exp \left[i a \frac{N(N+2)}{4}\right]\left|\Omega_{\phi, N}\right\rangle
\end{aligned}
$$

and there exists only one ground state for $H_{B C S}$.

The Bogoliubov ground state $\omega_{\phi}$ reflects the mean field theory and it contains the phase that becomes important for the Josephson effect. However it violates gauge symmetry. Only in the real ground state, i.e. in the coherent superposition of the Bogoliubov ground states the number of electrons (and therefore also of Cooper pairs) is preserved.

\section{The Macroscopic Algebra in the Ground State}

The main advantage to start with the BCS-Hamiltonian lies in the fact that it explains the thermodynamic structure with respect to phase transition and temperature dependence. But many aspects of superconductivity are explained by using wave functions on a macroscopic 
level. It is our aim to deduce these wavefunctions as corresponding to the expectation values of a suitable subset of observables that in addition have to be sufficiently independent of local fluctuations. Of course the BCS-Hamiltonian does not reflect the shape of the superconductor. Therefore we cannot expect that we will be able to find a subalgebra such that the corresponding wavefunction permits a geometric interpretation. Therefore we cannot give any contribution to the Ginzburg-Landau theory. But we are able to construct an algebra that has a strong similarity to the phenomenological Hamiltonian used to characterize the Josephson effect.

In [6] the fluctuation algebra was introduced. As typical example one can examine whether the following limit exists

$$
\hat{\omega}(\exp [i m(\vec{a} \vec{\sigma})])=: \lim _{N \rightarrow \infty} \omega_{N}\left(\exp \left[i \vec{a} \sum_{-N / 2}^{N / 2} \frac{\vec{\sigma}^{k}-\omega\left(\vec{\sigma}^{k}\right)}{\sqrt{N}}\right]\right)
$$

and allows an interpretation as state over a Weyl algebra built by operators $\exp [i m(\vec{a} \vec{\sigma})]$ that are characterized by the commutation relation, e.g.

$$
\begin{aligned}
& \hat{\omega}\left(\exp \left[\operatorname{iam}\left(\sigma_{x}\right)\right] \exp \left[\operatorname{ibm}\left(\sigma_{y}\right)\right]\right) \\
& \quad=\exp \left[-i a b s_{z}\right] \hat{\omega}\left(\exp \left[\operatorname{ibm}\left(\sigma_{y}\right)\right] \exp \left[\operatorname{iam}\left(\sigma_{x}\right)\right]\right),
\end{aligned}
$$

where $s_{z}=\omega\left(\sigma_{z}\right)$. Therefore $m\left(\sigma_{x}\right), m\left(\sigma_{y}\right)$ can be interpreted as properly scaled position and momentum operators. Adding $m\left(\sigma_{z}\right)$ it will depend linearly on $m\left(\sigma_{x}\right), m\left(\sigma_{y}\right)$. This procedure works if we take the limit $N \rightarrow \infty$ of $\omega_{\phi, N}$. Especially if we choose $\mu=0$ and $\phi=0$ where $\vec{S}$ shows in the $x$-direction, then (compare [11])

$$
\begin{aligned}
\lim \left\langle\psi_{0, N}\left|\frac{\sum \sigma_{y}^{k}}{\sqrt{N}}\right| \psi_{0, N}\right\rangle & =0, \\
\lim \left\langle\psi_{0, N}\left|\frac{\left(\sum \sigma_{y}^{k}\right)^{2}}{N}\right| \psi_{0, N}\right\rangle & =\frac{1}{4}, \\
\lim \left\langle\psi_{0, N}\left|\exp \left[i r \sum \sigma_{y}^{k} / \sqrt{N}\right]\right| \psi_{0, N}\right\rangle & =\exp \left[-\frac{r^{2}}{8}\right], \\
\lim \left\langle\psi_{0, N}\left|\exp \left[i r \sum \sigma_{z}^{k} / \sqrt{N}\right]\right| \psi_{0, N}\right\rangle & =\exp \left[-\frac{r^{2}}{8}\right] .
\end{aligned}
$$

We obtain a Weyl algebra over $L^{2}(R)$, built by $m\left(\sigma_{y}\right), m\left(\sigma_{z}\right)$, whereas $m\left(\sigma_{x}\right)$ is just a $c$-number. Varying $\phi$ the Weyl algebra will depend on the phase, but they all are isomorphic and the phase has no operational meaning. We can also calculate the time evolution. $m\left(\sigma_{z}\right)$ is 
invariant under the time evolution whereas $(d / d t) m\left(\sigma_{z}\right)=\omega\left(\sigma_{x}\right) m\left(\sigma_{z}\right)$ and is therefore the free time evolution for the Weyl algebra. Notice that the state is not invariant under this time evolution which indicates that the state does not have the desired stability properties.

If, however, we turn to the real ground state the fluctuations of $\sigma_{x}, \sigma_{y}$ are of macroscopic size so that the above limit does not exist. For $\sigma_{z}$ the coherent superposition fixes the fluctuations. Therefore we have to consider the following limits in a different scaling, namely:

$$
\begin{aligned}
& \hat{\omega}\left(\exp [ \pm i k \phi] \exp \left[\operatorname{iap}_{\phi}\right] \exp [ \pm i l \phi]\right) \\
& \quad=\lim \omega_{N}\left(\left(\frac{S_{ \pm}}{N c(\mu)}\right)^{k} \exp \left[i a \sum_{k}^{N}\left(\sigma_{z}^{k}-\omega_{N}\left(\sigma_{z}^{k}\right)\right)\right]\left(\frac{S_{ \pm}}{N c(\mu)}\right)^{l}\right) \\
& \quad=\exp [i \pi a( \pm l \pm k+\nu)] \delta_{k-l, 0} .
\end{aligned}
$$

The value $c(\mu)$ is adjusted so that

$$
\omega_{N}\left(\frac{S_{+}}{N c(\mu)} \frac{S_{-}}{N c(\mu)}\right)=1 .
$$

The value $\nu$ corresponds to the fact that the limit only exists for a subsequence of $N$ for which

$$
\left[\frac{N \mu}{2}\right]-\frac{N \mu}{2}=\nu
$$

Therefore we can interpret the limit in (3.3) as the expectation value over the Weyl algebra on the torus in the representation of the Weyl algebra where the boundary condition of $p_{\phi}$ is determined by the domain $\mathcal{D}\left(\left.p_{\phi}\right|_{\nu}\right)=\left\{\Psi \in L^{2}([0,2 \pi]), \lim _{\varepsilon \rightarrow 0} \Psi(\varepsilon)=\lim _{\varepsilon \rightarrow 0} e^{2 \pi i \nu} \Psi(2 \pi-\varepsilon)\right\}$.

So far we have succeeded to specify the noncommutative algebra in which the phase $\phi$ is imbedded. The time evolution of this algebra can be calculated by studying the commutator

$$
\begin{aligned}
{\left[H_{B C S}, S_{z}\right] } & =0, \\
\frac{N}{4}\left[H_{B C S}, \frac{S_{+}}{N}\right] & =i\left(\frac{S_{z}}{N}-\frac{\mu}{2}\right) \frac{S_{+}}{2}+i \frac{S_{+}}{2}\left(\frac{S_{z}}{N}-\frac{\mu}{2}\right) \rightarrow\left[p_{\phi}^{2}, e^{i \phi}\right] .
\end{aligned}
$$

Therefore the time evolution of the macroscopic phase algebra of an isolated superconductor is the free one on the torus, i.e. $\phi$ changes linearly in time. Of course we have to notice the different scaling, in the macroscopic phase algebra also the time has to be scaled to a macroscopic size. 


\section{Weakly Coupled Superconductors and the Josephson Effect}

Our next task is to relate this phase algebra to the Josephson effect. We assume that we have two superconductors that can be described by

$$
\begin{aligned}
H= & \sum_{k}^{N} \mu\left(a_{k}^{\dagger} a_{k}+b_{k}^{\dagger} b_{k}\right)-\frac{1}{N} \sum_{k, l}^{N} a_{l} b_{-l} b_{k}^{\dagger} a_{-k}^{\dagger}+\sum_{k}^{N} \bar{\mu}\left(c_{k}^{\dagger} c_{k}+d_{k}^{\dagger} d_{k}\right) \\
& -\frac{1}{N} \sum_{k, l}^{N} c_{l} d_{-l} d_{k}^{\dagger} c_{-k}^{\dagger} .
\end{aligned}
$$

The creation and annihilation operator $c^{\sharp}, d^{\sharp}$ belong to the second superconductor. Between the two superconductors there is a weak coupling that can be described as a perturbation of the Hamiltonian by

$$
\left.W=\gamma_{N} \sum_{k, l}\left(a_{k}^{\dagger}+b_{k}^{\dagger}\right)\left(c_{l}+d_{l}\right)+\text { h.c. }\right) \text {, }
$$

where we do not specify yet how $\lambda$ depends on $N$. JOSEPHSON $[1,12]$ treated the effect of this perturbation on the level of linear response theory. A current arises if the calculation is pursued to second order. This motivates us to replace the perturbation essentially by its squared so that the effect appears already in first order perturbation theory. In addition this enables us to calculate in $\sigma$-space. We describe the two superconductors by two sets of Pauli matrices, $\sigma^{k}, \tau^{k}$ and replace (4.1) by

$$
\begin{aligned}
H_{B C S}= & -\frac{4}{N} \sum_{k, l}^{N}\left(\sigma_{+}^{k} \sigma_{-}^{l}\right)+4 \sum_{k}^{N}\left(\mu-\frac{1}{N}\right) \sigma_{z}^{k}-\frac{4}{N} \sum_{k, l}^{N}\left(\tau_{+}^{k} \tau_{-}^{l}\right) \\
& +4 \sum_{k}^{N}\left(\bar{\mu}-\frac{1}{N}\right) \tau_{z}^{k} .
\end{aligned}
$$

Similarly we replace the perturbation (4.2) by

$$
W=\lambda_{N} 4 \sum_{k, l}\left(\sigma_{+}^{k} \tau_{-}^{l}+\sigma_{-}^{k} \tau_{+}^{l}\right) .
$$

The Josephson effect is considered to be expressed by a wave function. Therefore we choose the $N$-dependence of $\lambda$ in such a way that the perturbation reduces to an operator over $L^{2}(T, d \phi) \otimes L^{2}(T, d \psi)$ with $L^{2}(T, d \psi)$ the appropriate space for the second superconductor. This holds if we scale $\lambda_{N}=\lambda / N^{2}$, such that

$$
W \rightarrow c(\lambda, \mu, \bar{\mu})(\exp [i(\phi-\psi)]+\exp [-i(\phi-\psi)]) .
$$


In this scaling however the order of magnitude of the perturbation is larger than the energy difference so that perturbation theory is not justified. Therefore scaling like $\lambda_{N}=\lambda / N^{3}$ seems more appropriate. In this scaling the mesoscopic algebra over the phase space remains decoupled from the quasilocal algebra. The Hamiltonian on the phase space becomes

$$
H=\frac{1}{N}\left(p_{\phi}^{2}+p_{\psi}^{2}\right)+\frac{\lambda}{N} \cos (\phi-\psi)=\frac{1}{N}\left(p_{\phi+\psi}^{2}+p_{\phi-\psi}^{2}\right)+\frac{\lambda}{N} \cos (\phi-\psi) .
$$

Here we keep the $N$-dependence to indicate that kinetic energy and interaction are of the same order of magnitude. In order to interpret the effect of the Hamiltonian as time evolution we also have to scale time. The boundary conditions of $p_{\phi \pm \psi}$ are $\nu \pm \bar{\nu}$, the corresponding expressions of the individual boundary conditions for the two superconductors. A current arises as

$$
\begin{aligned}
J & =\frac{d}{d t}\langle Q\rangle=\frac{d}{d t}\left\langle\sum_{k}\left[\left(a_{k}^{\dagger} a_{k}+b_{k}^{\dagger} b_{k}\right)-\left(c_{k}^{\dagger} c_{k}+d_{k}^{\dagger} d_{k}\right)\right]\right\rangle \\
& =\left\langle i\left[p_{\phi}-p_{\psi}, V\right]\right\rangle=a\langle\sin (\phi-\psi)\rangle
\end{aligned}
$$

with some appropriate $a$ proportional to $\lambda$.

The main interest in the Josephson effect is of course the possibility that we are able to influence the current and that the way how it reacts can only be explained on the basis of quantum mechanics. Since in our model the geometry of the junction is not included the analysis of possible experimental setup is rather limited. However we can recover some of the main observations. If a constant voltage $V$ is applied across the Josephson junction then the current varies according to [13]

$$
J=J_{0} \sin \left(\phi+\frac{2 e}{h} V t\right)
$$

This formula is so accurate that it serves to define the standard volt, since the frequency only depends on the voltage [16]. We can recover the same time dependence if we relate the effect of the constant voltage to the mapping $a_{k} \rightarrow e^{i V t} a_{k}$ and this effect is transported to a constant shift on the torus, commuting with the momentum, i.e. it cannot create or annihilate Cooper pairs. What is essential is the independency on the coupling parameter in the perturbation or on other parameters entering in $H_{B C S}$. 
Since the Josephson junction is also used in squids, its quantum effects have been studied in detail. Here it is usually described by a Hamiltonian [15]

$$
H_{\text {box }}=4 E_{C}\left(n-\frac{Q_{g}}{2 e}\right)^{2}-E_{J} \cos \theta .
$$

"Here $n$ is the operator counting the number of excess Cooper pairs on the island relative to a neutral reference state. It is conjugate to $\theta$, the phase difference of the superconducting order parameter across the junction." The other parameters are interpreted in terms of gate charge and gate and junction capacities. We recognize our Hamiltonian (4.5) in the chosen scaling: In order that both terms, kinetic energy and potential are of the same order of magnitude we have scaled $\lambda_{N}$ as $\lambda / N^{3}$. Experimental adjustment indicates however that $E_{C} \ll E_{J}[14]$, which corresponds to large $\lambda / N^{3}$.

\section{The Phase Algebra at Finite Temperature}

The fact that the Josephson junction is so stable under variations of the setup asks, that we must also be able to vary the model. Especially we know that it shows only mild dependence on the temperature [17] away from the phase transition. Therefore also in equilibrium states at small temperature we must be able to construct the phase algebra and its states.

Again we consider as equilibrium state

$$
\omega_{N, \beta}(A)=\frac{\operatorname{Tr} \exp \left[-H_{B C S}\right] A}{\operatorname{Tr} \exp \left[-H_{B C S}\right]} .
$$

If we concentrate on operators $A$ that are finite sums of finite products in $\sigma$-matrices then it was shown in [3] that we can consider the limit $\omega_{\beta}(A)=\lim _{N} \omega_{N, \beta}(A)$ and obtain

$$
\omega_{\beta}(A)=\int_{0}^{2 \pi} d \phi \omega_{\beta, \phi}(A)
$$

where $\omega_{\beta, \phi}(A)$ similar as for the ground state is a product state that for a lattice point $k$ is given by

$$
\omega(\beta, \phi)\left(\vec{\sigma}^{k}\right)=\operatorname{Tr} \exp \left[-\beta\left(\vec{n}_{\beta, \phi} \vec{\sigma}\right)\right] \vec{\sigma} / \operatorname{Tr} \exp [-\beta(\vec{n}(\beta, \phi) \vec{\sigma})] .
$$

Here $\vec{n}(\beta, \phi)$ has to be determined by the self-consistency equation

$$
n_{x}(\beta, \phi)=\omega_{\beta, \phi}\left(\sigma_{x}\right), \quad n_{y}(\beta, \phi)=\omega_{\beta, \phi}\left(\sigma_{y}\right), \quad n_{z}(\beta, \phi)=\mu .
$$


$\omega_{\beta, \phi}$ can be interpreted as equilibrium state because $\exp \left[i t H_{B C S}\right] \vec{\sigma} \times$ $\times \exp \left[-i t H_{B C S}\right]$ converges strongly in the GNS representation corresponding to $\omega_{\beta, \phi}$ to $\left.\exp [i t(\vec{n}(\beta, \phi) \vec{\sigma})] \vec{\sigma} \exp [-i t \vec{n}(\beta, \phi) \vec{\sigma})\right]$ and $\omega_{\beta, \phi}$ satisfies the KMS condition with respect to this effective time evolution. However it is not gauge invariant and the particle number is not preserved. This is cured in the integrated version $\omega_{\beta}$. But this integrated version does not yet specify the expectation value of mesoscopic observables.

In [3] the state was calculated by estimating the fluctuation of $S^{2} / N$ and $S_{z}$. For both operators the fluctuations are of the order $\sqrt{N}$. Therefore we cannot obtain a limit for $S_{z}-\omega_{\beta}\left(S_{z}\right)$ serving as conjugate variable to the phase $\phi$. For $S_{+}$the fluctuations are of macroscopic size and we have to change the scaling as we did for the ground state. In this scaling we have to examine

$$
\lim _{\rightarrow \infty} \operatorname{Tr} \exp \left[-\beta H_{N}\right] \exp \left[i a S_{z}\right]\left(\frac{S_{+}}{N}\right)^{k} / \operatorname{Tr} \exp \left[-\beta H_{N}\right] .
$$

We can expand in eigenfunctions of $S, S_{z}$ so that the limit vanishes if $k \neq 0$. For $k=0, a \neq 0$ it is not defined and we have to control the fluctuations of $S_{z}$ by a renormalization procedure. In order to make this renormalization procedure explicit to obtain the limit $S_{+} / N$ as an operator over $L^{2}(T)$ we construct a sequence of states that gives the same limit for local (in $K$ ) operators, becomes a pure state for an appropriate macroscopic algebra, but in addition is similar to the coherent superposition for the ground state (2.5). We consider the states $\omega_{\beta, \phi}$ for finite $N$. They can be represented by a vector $\left|\Omega_{\beta, \phi}, N\right\rangle$ in a GNS representation. This representation is unique up to an identification of the commutator. This identification is possible, because the states are related by a unitary transformation, as long as we keep $N$ finite. We can write

$$
\left|\Omega_{N}\right\rangle=\int_{0}^{2 \pi} d \phi \otimes\left|\sqrt{\rho_{\phi}} U_{\phi}\right\rangle
$$

Here $\rho_{\phi}$ is the density operator for the lattice point and $U_{\phi}$ is a unitary operator that we can adjust in order to specify the coherent superposition of the states, but it has no physical meaning. The state implemented by $\left|\Omega_{N}\right\rangle$ is represented in its GNS representation, because we have a pure state over the observable algebra together with its commutant, and the commutant is antiisomorphic to the observable algebra. As a consequence of the integration we obtain a state that on the local level is invariant under rota- 
tion implemented by $\exp \left[i a S_{z}\right]$. As for the ground state we study the effect

$$
\exp \left[i a S_{z}\right]\left|\Omega_{\beta, N}\right\rangle=\int_{0}^{2 \pi} d \phi \otimes\left|\sqrt{\rho_{\phi+a}} \exp \left[i a \sigma_{z}\right] U_{\phi}\right\rangle .
$$

It is impossible to find a $U_{\phi}$ so that the $a$-dependence is covered by the integration. We have to consider

$$
\exp \left[i a\left(S_{z}-S_{z}^{\prime}\right)\right]\left|\Omega_{\beta, N}\right\rangle=\int_{0}^{2 \pi} d \phi \otimes\left|\sqrt{\rho_{\phi+a}}\right\rangle=\left|\Omega_{\beta, N}\right\rangle .
$$

$S_{z}^{\prime}$ is the operator in the commutant that corresponds to multiplication from the right in the representation for finite dimensions. That we need it to obtain convergence corresponds to the fact that the rotation around the $z$-axes is not an inner automorphism but only approximately inner. This enables us to get

$$
\begin{aligned}
& \hat{\omega}_{\nu}\left(\exp [ \pm i k \phi] \exp \left[i a_{\phi}\right] \exp [ \pm i l \phi]\right) \\
& \quad=\lim \left\langle\Omega_{\beta}, N\left|\left(\frac{S_{ \pm}}{N c(\mu)}\right)^{k} \exp \left[i a \sum_{k}^{N}\left(\sigma_{z}^{k}-\sigma_{z}^{k \prime}\right)\right]\left(\frac{S_{ \pm}}{N c(\mu)}\right)^{l}\right| \Omega_{\beta}, N\right\rangle \\
& \quad=\exp [i \pi a( \pm l \pm k)] \delta_{l-k, 0} .
\end{aligned}
$$

The algebra changes from the one corresponding to the ground state by adjusting the correction value by an operator in the commutant that absorbs the fluctuations of $S_{z}$. In addition we have to adjust also the normalization factor $c(\mu)$. There is one further important difference: For the ground state we could justify that we choose a special value $\nu$ by choosing an appropriate sequence of $N$. Now the variable conjugated to the phase is not an observable that can be obtained in a limiting procedure from observables and therefore has no operational interpretation. Therefore it is natural to choose $\nu=0$, so that the limiting state on the mesoscopic algebra is the ground state for the Hamiltonian $h=p_{\phi}^{2}$. But this Hamiltonian does not characterize the time evolution of $e^{i \phi}$. As for the ground state we have

$$
\begin{aligned}
{\left[H_{B C S}, S_{z}\right] } & =0, \\
N\left[H_{B C S}, \frac{S_{+}}{N}\right] & =i \frac{S_{z} S_{+}}{N}+i \frac{S_{+} S_{z}}{N}-i \mu N \frac{S_{+}}{N}=\frac{S_{+}}{N}\left(1+2 S_{z}-\mu N\right) .
\end{aligned}
$$

Therefore we have no strong convergence. Of course there is also an alternative construction of the limiting state for the quasilocal algebra: We can start as in [3] with a state as sum of eigenstates for the total 
angular momentum $S^{2}$ and $S_{z}$, but with $S_{z}$ fixed to the value dictated by the limiting state. Then no renormalization with operators of the commutant is necessary and the final results correspond to those in the ground state, though the coherent superposition is somewhat hidden. But since the expectation value of $\sigma_{z}$ is a consequence of the interplay of energy and entropy and depends on the temperature and allows fluctuations in the energy, it seems hard to justify such a restriction.

We return to the Josephson current between two superconductors and we keep the coupling $\lambda_{N}=\lambda / N^{3}$. For this very weak coupling it was shown in [4] that on the level of the quasilocal algebra the state converges to

$$
\omega=\int d \gamma_{1} d \gamma_{2} \otimes_{k} \omega_{\gamma_{1}}^{k} \otimes_{l} \omega_{\gamma_{2}}^{l},
$$

so that in the bulks all states at different lattice points are in the same phase, but the phases of the two bulks are uncorrelated. This state can however be obtained as limit of

$$
\int_{0}^{2 \pi} d \phi d \psi N^{-2} \sum_{k l} \otimes\left|\sqrt{\rho_{\phi, \psi, k, l}}\right\rangle,
$$

where we have entangled randomly the state of one superconductor at position $k$ in orientation $\phi$ with the state of the other superconductor at position $l$ in orientation $\psi$ in the sense that

$$
\vec{\sigma} \otimes \vec{\tau}\left|\sqrt{\rho_{\phi, \psi}}\right\rangle=\left|\vec{\sigma} \sqrt{\rho_{\phi}} \exp [-i(\phi-\psi)] \vec{\tau}^{t}\right\rangle
$$

where $\vec{\tau}^{t}$ is the transposed. Now we can proceed to construct the phase algebra in the sense of (5.2) with the difference that

$$
\exp \left[i a\left(\sigma_{k}^{z}-\tau_{l}^{z}\right)\right]\left|\sqrt{\rho_{\phi}} \exp [-i(\phi-\psi)]\right\rangle=\left|\sqrt{\rho_{\phi+a}} \exp [-i(\phi-\psi)]\right\rangle
$$

already implies

$$
\exp \left[i a\left(S_{z}-T_{z}\right)\right]\left|\Omega_{\beta, N}\right\rangle=\left|\Omega_{\beta, N}\right\rangle .
$$

Therefore we are let to a Hamiltonian as in (4.5):

$$
\begin{aligned}
H & =\frac{1}{N}\left(p_{\phi}^{2}+p_{\psi}^{2}\right)+\frac{\lambda}{N} \cos (\phi-\psi) \\
& \left.=\frac{1}{N}\left(S_{z}+T_{z}-\mu N-\nu N\right)^{2}+p_{\phi-\psi}^{2}\right)+\frac{\lambda}{N} \cos (\phi-\psi) .
\end{aligned}
$$

We can ignore the first term and concentrate on $\phi-\psi$. We realize that the Josephson current is not expressed by the momentum but 
by the commutator of the momentum with a function of the phase difference. For this commutator no regularization with operators from the commutant are necessary due to our choice of convergence of the states: In the limit the local fluctuations of $\sigma_{z}^{k}$ cancel with those of the other bulk. Only the bulk property of $p_{\phi-\psi}$ remains responsible for the Josephson current and the time evolution of the phase algebra. Compare also the considerations in [5] where it is also argued that oscillating terms have to be thrown out. Corresponding consideration hopefully justifies the choice of the states (5.7). We have to remember that the actual interaction between the two superconductors couples electrons and not Cooper pairs. As in the construction in [12] the change of the state has to be taken into account to obtain in second order perturbation theory the Josephson current. Different from the number of Cooper pairs the number of electrons is a preserved quantity. Therefore it is not hopeless but seems to be a delicate question that the interaction courses the desired entanglement between the two bulks.

Having justified the mesoscopic algebra on the basis of this special sequence of states two superconductors in weak contact can now be treated in total analogy as for the ground state. The temperature only effects the scaling of $\lim c^{-1}(\mu, \beta)\left(S_{+} / N\right)$ and therefore the strength of maximally possible $J_{0}$ in the Josephson current. Notice that the state reduced to the mesoscopic algebra $\left\{p_{\phi-\psi}, \exp [i(\phi-\psi)]\right\}$ remains the ground state and does not reflect the temperature. This is not in contradiction to the analyticity properties of the Gibbs state for finite $N$, because our limit procedure includes non-physical values for which the analyticity properties do not hold.

It remains to argue about the boundary condition of $p_{\phi-\psi}$. For the ground state it was natural that we expected the state to be pure. The boundary condition could be related to a special sequence in the limiting procedure. Now we consider the limit of mixed states. $p_{\phi}$ is defined as the variable conjugated to $e^{i \phi}=\lim S_{+} / N c(\mu)$ and only related to $S_{z}$. The boundary condition $\nu=0$ appears, if we demand that the system is in its ground state together with $\lim \omega\left(S_{+} / N\right)=0$. However in [15] the Hamiltonian appears with different boundary condition determined by a gage voltage. We know that the fluctuations of $S_{z}$ are of the order $\sqrt{N}$. These fluctuations cancel with an appropriate regularization that represents the macroscopic bulk. But finite fluctuations corresponding to different boundaries respectively to the fact that the mesoscopic system is not in its ground state do not cost so much energy that it would be in contradiction with the 
possible fluctuations of the total energy. Therefore we could expect as state over our phase algebra

$$
\hat{\omega}\left(\exp \left[\operatorname{iap}_{\phi}\right] \exp [i k \phi]\right)=\int_{0}^{1} d \nu \hat{\omega}_{\nu}\left(\exp \left[i a p_{\phi}\right] \exp [i k \phi]\right)
$$

or even mixed states for fixed $\nu$. Remember that we started from Gibbs states with some temperature. Why do we observe for the mesoscopic algebra only pure states, but not a unique one, whereas we started with a mixed Gibbs state?

If we are concerned with different thermodynamic phases on the macroscopic level then mathematically this corresponds to the fact that we can combine the different states in a classical superposition. This state leads in the corresponding GNS construction to a Von Neumann algebra with nontrivial center. This center is elementwise invariant under the time evolution. Decomposing the state with the unique central decomposition we obtain the pure phases. Considering the limit of Gibbs states for the increasing system we obtain, if necessary by choosing a subsequence, some superposition of these phases, which superposition might depend on the chosen subsequence or on the variation of boundary conditions. Especially if we vary the Hamiltonian and let the perturbation tend to 0 we can produce the pure thermodynamical phases. In our situation these perturbations have to violate gauge symmetry and therefore are not permitted. On the level of the quasilocal algebra the only acceptable equilibrium state is the gauge invariant state. But for the phase algebra the fluctuations of $S_{z}$ of order $\sqrt{N}$ always dominate over fixing the boundary condition for $p_{\phi}$. Appropriate perturbations cannot justify the fixing of $\nu$. Notice that for one bulk $\nu=0$ is necessary in order that the state is gauge invariant and therefore $\hat{\omega}\left(e^{i \phi}\right)=0$. For two bulks $e^{i \phi} e^{-i \psi}$ is gauge invariant and therefore all boundary values for the conjugated momentum are permitted, corresponding to the possibility that Cooper pairs can move between the bulks. Variation of the boundary condition does only cost finite amount of energy. Why do we not observe a statistical distribution of the boundary value?

In $[18,19]$, we tried to justify the fact that we observe pure thermodynamical phases in nature and not classical superpositions of them by a sequence of measuring processes. In every measuring process the state is changed to a new state in which the measured quantity has no fluctuations, otherwise the state remains as less influenced as possible. Therefore especially the state over the center of the representation is changed, if only the measured object has different expectation values in the different pure thermodynamical 
phases. Time evolution may sweep out the effect of the measurement, but it cannot sweep out the effect in the center, since the center is time invariant. Repeating measurements let us move in the center, we cannot predict how, because the outcome of the measurement is determined by probability theory, but this probability theory guarantees that with probability 1 we approach after infinitely many measurements a pure thermodynamical phase.

If we apply the same consideration to our present situation then measuring $p_{\phi-\psi}$ once fixes $\nu$. Time evolution (3.4) reduces on $\left\{p_{\phi-\psi}, e^{i(\phi-\psi)}\right\}$ to an inner evolution of the algebra and cannot change $\nu$. Also local measurements, i.e. local changes of the state cannot influence $\nu$. This makes the reduction of any mixed state stable and permits us to treat $\left\{p_{\phi-\psi}, e^{i(\phi-\psi)}\right\}$ as an individual algebra in a pure state. Variation of the boundary condition $\nu$ becomes possible but only by an outer automorphism i.e. by an external voltage. This external voltage implements also an automorphism on the observable algebra for finite $N$ without effecting the commutant but reduces to an outer automorphism on the mesoscopic algebra.

\section{Strongly Coupled Superconductors}

We have already varied the model to include the way how the Josephson effect depends on the temperature. In addition we want to modify it in a way that enables us in a limited way to characterize the underlying geometry of the model. For instance in a typical example we have just one superconductor that forms a tube, which however is interrupted along a surface by a thin dielectric layer that permits a Josephson current. In order to mimic this situation we take a Hamiltonian

$$
H_{B C S}=\sum_{j=1}^{j=K}\left[4 \mu_{j} S_{z}^{j}-\frac{4}{N} S_{+}^{j} S_{-}^{j}\right]-\frac{1}{N} \sum_{j, \bar{j}} \lambda_{j, \bar{j}} S_{+}^{j} S_{-}^{\bar{j}} .
$$

The idea for this ansatz is that we split the superconductor into a finite number of $K$ bulks. The parameter $N$ now indicates the size of the bulks, and the interaction between different bulks is decreasing. If we assume that we have only one superconductor, $\mu_{j}=\mu$ has to be chosen independently of the bulk, but we can just as well make it $j$ dependent.

The ground state of this system should be calculated according to our considerations for $K=1$ by writing $H_{B C S}$ as function of $S_{j}^{2}, S_{z}^{j}, j=1, \ldots, K$. These operators commute with $H_{B C S}$, but they do 
not suffice to express it. Instead we use again the mean field structure of the Hamiltonian and consider again as in (2.5), (5.2)

$$
\left|\Omega_{\phi, N}\right\rangle=\otimes_{k=1}^{K}\left(\otimes_{j=1}^{N}\left|\alpha_{k}, \phi\right\rangle\right) .
$$

The state is a product state. How the spins are oriented in the $(x, y)$ space depends on $\phi$, independent to which bulk they belong. But they can be differently oriented in the $(x, z)$ space (where we choose $\phi=0)$ for different bulks. The orientation in the $(x, z)$ space (again with $\phi=0$ ) is given by minimizing

$\left\langle\Omega_{0, N}\left|\frac{4 H_{B C S}}{N}\right| \Omega_{0, N}\right\rangle=\sum_{k}^{K} \mu_{k} \cos \alpha_{k}-\sum_{j} \sin \alpha_{j}^{2}-\sum_{j, k}^{K} \lambda_{j, k} \sin \alpha_{j} \sin \alpha_{k}$,

which corresponds to the solution of

$$
2 \cos \alpha_{k} \sin \alpha_{k}+\sum_{j} \lambda_{k, j} \cos \alpha_{k} \sin \alpha_{j}-\mu_{k} \sin \alpha_{k}=0 .
$$

From this Bogoliubov state we can again pass to the ground state of $H_{B C S}$,

$$
\left|\Omega_{N}\right\rangle=\left|\int_{0}^{2 \pi} d \phi \Omega_{\phi, N}\right\rangle
$$

This state is an eigenstate of $H_{B C S}$ for the following reason: The operators $\sum_{j} S_{z}^{j}, S^{j 2}$ commute with $H_{B C S} .\left|\Omega_{N}\right\rangle$ is an eigenvector for these operators and since it maximizes the eigenvalue of $S^{j 2}$ it is the unique eigenvector with the same eigenvalues. Therefore it is invariant under the action of any operator that commutes with $\sum_{j} S_{z}^{j}, S^{j 2}$ and especially under $H_{B C S}$. That it is the ground state is a consequence of the minimization.

As before we can therefore construct the mesoscopic algebra as

$$
\lim \exp \left[i \gamma \sum_{k=1}^{K}\left(S_{z}^{k}-S_{z}^{k \prime}\right)\right]=\exp \left[i \gamma p_{\phi}\right] \lim \frac{S_{+}^{k}}{N c\left(\alpha_{k}\right)}=e^{i \phi} .
$$

Notice that for $\phi$ we have no dependence on the bulk. This follows because the limits of $S_{+}^{k}$ are not independent but satisfy

$$
\lim \frac{S_{+}^{j} S_{-}^{k}}{N^{2} c\left(\alpha_{j}\right) c\left(\alpha_{k}\right)}=1 .
$$

Therefore the mesoscopic algebra remains the Weyl algebra on the one-dimensional torus and does not reflect the number of bulks. 
We have, however, the possibility to construct additional mesoscopic algebras that reflect the fluctuations between the bulks. As in the usual construction of the fluctuation algebra [6] we take the limits

$\lim \left\langle\Omega_{N}\left|\exp \left[i \gamma \frac{S_{z}^{j}-N \cos \alpha_{j} / 2}{\sqrt{N}}\right]\right| \Omega_{N}\right\rangle=\exp \left[-i \gamma^{2} / 4\right]$,
$\lim \left\langle\Omega_{N}\left|\exp \left[i \gamma \frac{\sum_{k \in \Delta_{j}}^{N}\left(\sigma_{+}^{k} \sigma_{-}^{k+1}-\omega\left(\sigma_{+}^{k} \sigma_{-}^{k+1}\right)\right)}{\sqrt{N}}\right]\right| \Omega_{N}\right\rangle=\exp \left[-\gamma^{2} \sin \alpha_{j}^{2}\right]$.

These operators commute according to

$$
\lim \sum_{k, l} \frac{1}{N}\left[\sigma_{+}^{k} \sigma_{-}^{k+1}, \sigma_{z}^{l}\right]=0 .
$$

In order to get non-commuting mesoscopic observables we can take $\lim \left\langle\Omega_{N}\left|\exp \left[i \gamma \frac{\sum_{k}^{N}\left(\sigma_{+}^{k} \tau_{-}^{k}-\omega\left(\sigma_{+}^{k} \tau_{-}^{k}\right)\right)}{\sqrt{N}}\right]\right| \Omega_{N}\right\rangle=\exp \left[-\gamma^{2} \sin \alpha_{j} \sin \alpha_{l}\right]$, where $\sigma_{+}^{k}$ belongs to the set $\Delta_{j}$ and $\tau_{-}^{k}$ to the set $\Delta_{l}$. Now in the appropriate topology for the fluctuation algebra

$$
\lim \sum_{k, r} \frac{1}{N}\left[\sigma_{+}^{k} \tau_{-}^{k}, \sigma_{z}^{r}\right]=\lim \sum_{k} \frac{1}{N} \sigma_{+}^{k} \tau_{-}^{k}=\sin \alpha_{j} \sin \alpha_{l} .
$$

This additional mesoscopic algebra commutes with the quasilocal algebra as well as with the phase algebra and cannot influence the Josephson current.

Turning to temperature states we can again construct a sequence of Bogoliubov states coherently superposed in the GNS representation. However we can include a further variation and study its influence on the Josephson current. We know that in a temperature state we have to be aware of fluctuations under which the expectation value of the energy varies as $\pm \sqrt{N}$. Such a fluctuation can be obtained if we take as states a mixture

$$
\omega=\int_{\Delta} d^{K} \delta_{j} \omega_{\delta}
$$

with

$$
\Omega_{\delta}=\int_{0}^{2 \pi} d \phi \otimes_{j}\left|\Omega_{\phi+\delta_{j}}\right\rangle
$$


As long as $\Delta$ has diameter $1 / \sqrt{N}$ also the energy only fluctuates in the permitted region. Again we notice that $\lim S_{+}^{j} / N$ exists as before as well as $\lim \exp \left[i \gamma \sum_{j} S_{z}^{j}\right]$. Only the fixing of the boundary condition becomes more delicate. If it depends on $\delta$ then the integral over $\delta$ reduces for the phase algebra to an integral over the boundary condition, an effect that would smear out the periodicity dependence in (4.6). But we already realized that we have to find arguments that go beyond a limiting procedure to justify that our phase algebra is irreducibly represented. Regularization with the counterpart of the commutant stabilizes the boundary condition and disappears in commutators.

\section{Conclusion}

We constructed in the framework of the BCS-model a mesoscopic algebra that reflects the possible states for the Josephson current. The model cannot reflect the exact geometry of the superconductors and especially not the surface character of the Josephson junction. But we have shown that it is stable under variations, especially under a combination of mean field theory and local interaction, so that in a more detailed analysis it should permit to include the geometry of the junction and also permit to couple in a more realistic way the size of gage capacitance and junction capacitance (compare [15, 14]). However we have also pointed out that the fact that we are confronted with a mesoscopic pure state on this algebra cannot directly be deduced by a limiting procedure from Gibbs states. Also on the mesoscopic algebra we are confronted with a phase transition, but now the phase transition is with respect to boundary conditions, i.e. conjugated to the phase that is broken in the Bogoliubov states but not in the physical states.

\section{References}

[1] Josephson, B. D. (1962) Phys. Lett. 1: 251; (1964) Rev. mod. Phys. 36: 216

[2] Thirring, W., Wehrl, A. (1967) Commun. Math. Phys. 4: 303

[3] ThIRring, W. (1968) Commun. Math. Phys. 7: 181-189

[4] Ullrich, M. (1986) Rep. Math. Phys. 23: 67-81

[5] Unnerstall, T., RiecKers, A. (1992) Phys. Rev. B45/17: 10115

[6] Goderis, D., Verbeure, A., Vets, P. (1989) Prob. Th. Rel. Fields 82: 527; (1990) Commun. Math. Phys. 128: 533; (1991) Il Nuovo Cim. 106B: 375

[7] NARnhofer, H. (2004) Found. Phys. Lett. 17: 235-255

[8] Martin, Ph. A., Rothen, F. (2004) Many-Body Problems and Quantum Field Theory. An Introduction, 2nd Ed. Springer, Berlin Heidelberg

[9] Verbeure, A., Zagrebnov, V. A. (1992) J. Stat. Phys. 69: 329

[10] SEwell, G. (2002) Quantum Mechanics and Its Emergent Macrophysics. Princeton Univ. Press, Princeton and Oxford 
[11] IliEVA, N., NARNHOFER, H., ThIRRING, W.: quant-ph/0502100

[12] LEVY, L.-P. (2000) Magnetism and Superconductivity. Springer, Berlin

[13] Remoissenet, M. (1996) Waves Called Solitons, Concepts and Experiments. Springer, Berlin

[14] Friedman, J. R., Patel, V., Chen, W., Tolpygo, S. K., Lukens, J. E. (2001) In: Macroscopic Quantum Coherence and Quantum Computing (AverIn, D. V., Ruggiero, B., Silvestrini, P., eds.). Kluwer, New York

[15] Makhlin, Yu., Schoen, G., Shnirman, A. (2001) Rev. Mod. Phys. 73: 357-400; cond-math/0308297

[16] http://hyperphysics.phy-astr.gsu.edu/hbase/solids/squid.html

[17] Ambegaokar, V., Baratoff, A. (1963) Phys. Rev. Lett. 10/11: 486; (1963) Phys. Rev. Lett. 11: 104

[18] NARnhofER, H. (1993) In: Phase Transitions (KoteCKI, R., ed.), pp. 150-158. World Scientific, Singapore

[19] NARnhofer, H., Thirring, W. (1999) In: On Quanta, Mind and Matter (AtTmanspacher, H., Amann, A., Mueller-Herold, U., eds.), pp. 105-118. Kluwer, Dordrecht

Author's address: A.o. Univ.-Prof. Dr. Heide Narnhofer, Institut für Theoretische Physik, Universität Wien, Währinger Straße 38-42, 1090 Wien, Austria. E-Mail: heide.narnhofer@univie.ac.at. 